\title{
A unique de novo gain-of-function variant in CAMK4 associated with intellectual disability and hyperkinetic movement disorder
}

\author{
Michael Zech, ${ }^{1,2,8}$ Daniel D. Lam, ${ }^{1,8}$ Sandrina Weber, ${ }^{1}$ Riccardo Beruttii, ${ }^{3}$ \\ Kamila Poláková, ${ }^{4}$ Petra Havránková, ${ }^{4}$ Anna Fečíková, ${ }^{4}$ Tim M. Strom, ${ }^{3,5}$ \\ Evžen Rǔžička, ${ }^{4}$ Robert Jech, ${ }^{4,9}$ and Juliane Winkelmann ${ }^{1,5,6,7,9}$
}

Corresponding author: juliane.winkelmann@tum.de

(C) 2018 Zech et al. This article is distributed under the terms of the Creative Commons Attribution License, which permits unrestricted reuse and redistribution provided that the original author and source are credited.

Ontology terms: athetoid cerebral palsy; language impairment; motor deterioration; psychomotor deterioration: torticollis

Published by Cold Spring Harbor Laboratory Press

doi: $10.1101 /$ mcs.a003293
Abstract Calcium/calmodulin-dependent protein kinases (CaMKs) are key mediators of calcium signaling and underpin neuronal health. Although widely studied, the contribution of CaMKs to Mendelian disease is rather enigmatic. Here, we describe an unusual neurodevelopmental phenotype, characterized by milestone delay, intellectual disability, autism, ataxia, and mixed hyperkinetic movement disorder including severe generalized dystonia, in a proband who remained etiologically undiagnosed despite exhaustive testing. We performed trio whole-exome sequencing to identify a de novo essential splice-site variant (c.981+1G $>A$ ) in CAMK4, encoding CaMKIV. Through in silico evaluation and cDNA analyses, we demonstrated that c. $981+1 \mathrm{G}>\mathrm{A}$ alters CAMK4 pre-mRNA processing and results in a stable mRNA transcript containing a 77-nt out-of-frame deletion and a premature termination codon within the last exon. The expected protein, p.Lys303Serfs*28, exhibits selective loss of the carboxy-terminal regulatory domain of CaMKIV and bears striking structural resemblance to previously reported synthetic mutants that confer constitutive CaMKIV activity. Biochemical studies in proband-derived cells confirmed an activating effect of c.981+1G>A and indicated that variant-induced excessive CaMKIV signaling is sensitive to pharmacological manipulation. Additionally, we found that variants predicted to cause selective depletion of CaMKIV's regulatory domain are unobserved in diverse catalogs of human variation, thus revealing that c. $981+1 \mathrm{G}>\mathrm{A}$ is a unique molecular event. We propose that our proband's phenotype is explainable by a dominant CAMK4 splice-disrupting mutation that acts through a gain-of-function mechanism. Our findings highlight the importance of CAMK4 in human neurodevelopment, provide a foundation for future clinical research of CAMK4, and suggest the CaMKIV signaling pathway as a potential drug target in neurological disease.

[Supplemental material is available for this article.]

\footnotetext{
${ }^{8}$ These authors contributed equally to this work as first authors.

${ }^{9}$ These authors contributed equally to this work as senior authors.
} 


\section{INTRODUCTION}

Calcium $\left(\mathrm{Ca}^{2+}\right)$ signaling is of vital importance to human physiology and plays diversified roles in biological and pathological cerebral functions (Rosenberg and Spitzer 2011; Bading 2013). Within the $\mathrm{Ca}^{2+}$-driven biochemical machinery, the $\mathrm{Ca}^{2+}$ /calmodulin-dependent serine/threonine protein kinases (CaMKs) are a class of master signaling effectors critically involved in various stages of neural development, including proliferation, migration, differentiation, and survival (Penzes et al. 2008; Takemoto-Kimura et al. 2017). In the mammalian brain, there are at least five known CaMKs: CaMKI, CaMKII, CaMKIII, CaMKIV, and CaMK kinase (CaMKK) (Swulius and Waxham 2008). Among these, CaMKIV is widely expressed in telencephalon and cerebellum, where it is predominantly localized to cell nuclei and orchestrates synapse-to-nucleus communication via determination of transcriptional outcomes (Jensen et al. 1991; Bading 2013). Human CaMKIV, encoded by CAMK4 on Chromosome $5 q 22.1$, is a monomeric 473-amino acid enzyme and subdivided into two main functional domains (Tokumitsu et al. 1997; Anderson et al. 2004; Naz et al. 2016): a protein kinase domain at the amino terminus (residues 46-300) and an autoregulatory domain at the carboxyl terminus (residues 305-341). The autoregulatory domain contains an autoinhibitory domain (AID) (residues 305-321), immediately followed by a calmodulin-binding domain (CBD) (residues 322-341), and forms a binding site for protein phosphatase 2A (PP2A). Because of its crucial involvement in $\mathrm{Ca}^{2+}$-dependent neuronal integrity, CaMKIV catalytic function is tightly controlled. In particular, unregulated autonomous activity of CaMKIV is prevented by molecular mechanisms involving its autoregulatory domain: (1) in the absence of $\mathrm{Ca}^{2+}$ stimulation, the AID blocks the catalytic core of the kinase domain, thereby maintaining CaMKIV in an autoinhibited state; (2) for release of autoinhibition, CaMKIV must bind an active $\mathrm{Ca}^{2+} /$ calmodulin complex, requiring proper interaction with the CBD; and (3) following activation, CaMKIV is subject to rapid enzymatic deactivation, mediated by an association of the autoregulatory sequence with PP2A (Tokumitsu et al. 1997; Anderson et al. 2004; Naz et al. 2016).

Although CaMKs have attracted widespread research interest, the only indication that these enzymes are connected to human brain disease comes from the very recent identification of heterozygous dominant mutations in CAMK2A/CAMK2B (the genes encoding subunits of CaMKII) in individuals with nonsyndromic intellectual disability (Küry et al. 2017). Whether perturbation of other members of the CaMK family also causes Mendelian disease was still unknown. In this article, we report a second CaMK-related neurodevelopmental disorder. We describe a proband presenting with an unusual phenotype comprising intellectual disability and progression to severe movement disorder, who was found to harbor a germline de novo essential splice-site variant in CAMK4. We demonstrate that the splicing alteration results in heterozygous expression of CaMKIV with a truncated autoregulatory domain, mimicking in vitro-engineered deletion mutants that have been used for decades in the study of constitutive CaMKIV activation.

\section{RESULTS}

\section{Clinical Presentation and Diagnostic Investigation}

We encountered a 28-yr-old male proband of European descent (proband A-4 in family A; see Fig. 2A) who was referred to the Department of Neurology and Center of Clinical Neuroscience at Charles University in Prague (Czech Republic) because of neurodevelopmental disability and generalized hyperkinetic movements (Table 1; Fig. 1). Prior to diagnostic and research investigations, written informed consent was obtained from the proband 


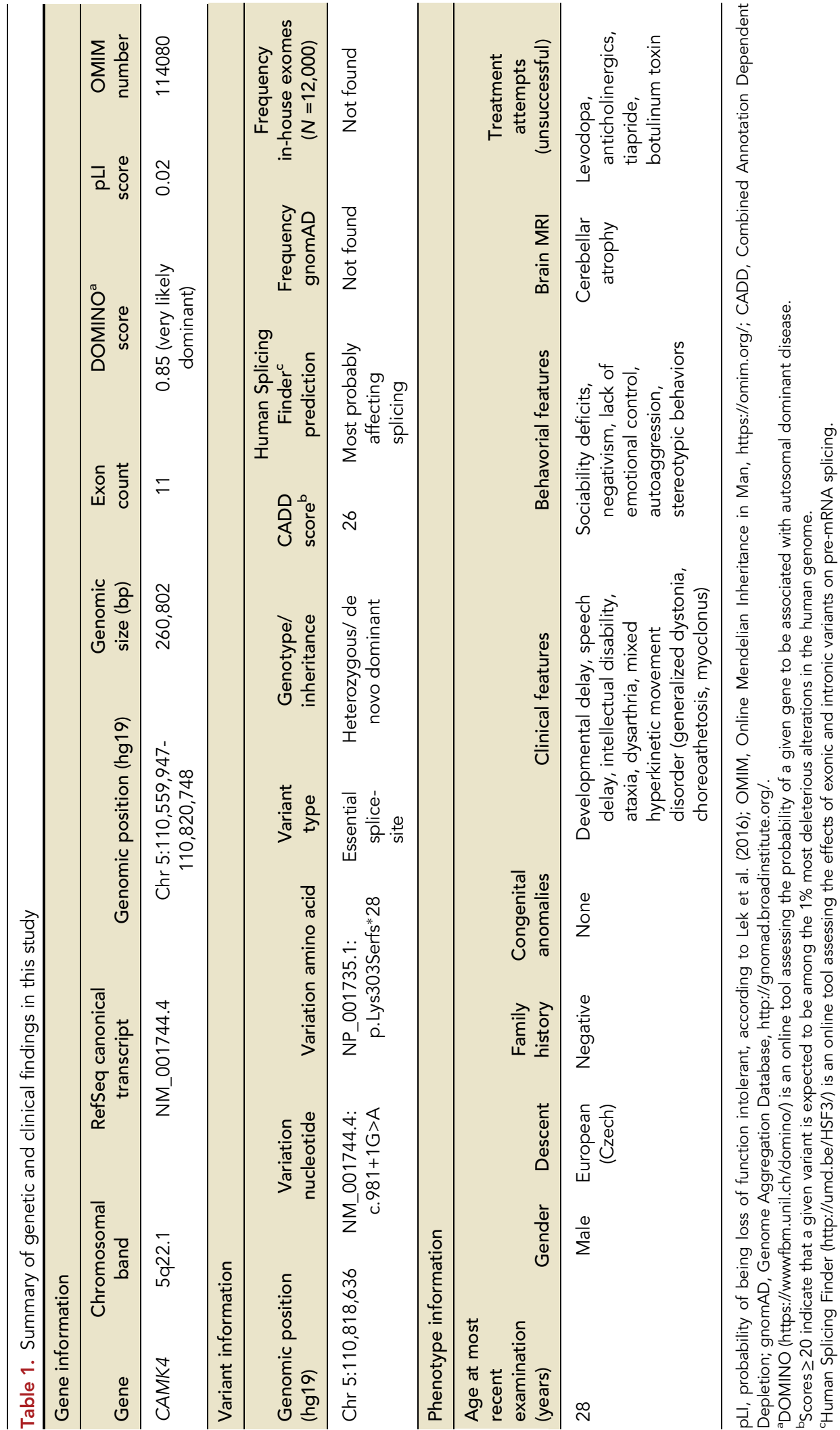


COLD SPRING HARBOR Molecular Case Studies
CAMK4 variant in neurodevelopmental motor disease
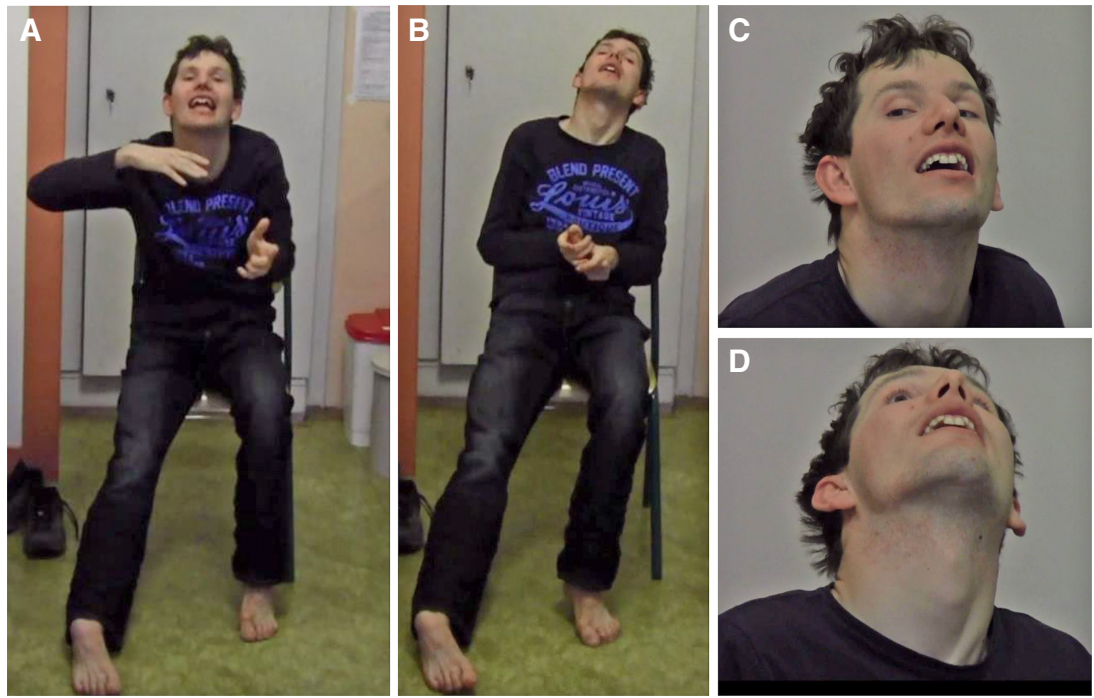

Figure 1. Pictures of proband A-4 with a de novo variant in CAMK4. Photographs of proband $A-4$ at the age of $28 \mathrm{yr}$ showing his whole body $(A, B)$ and close-ups of his face $(C, D)$. Note involuntary abnormal postures.

and his family members in accordance with standard protocols approved by local ethics review boards. Proband A-4 was the second child of healthy nonconsanguineous parents, aged 27 (mother, A-2 in Fig. 2A) and $29 \mathrm{yr}$ (father, A-1 in Fig. 2A) at the time of conception. His older sister (A-3 in Fig. 2A) was healthy. There was no family history of neurological or neurodevelopmental disease. Although his mother suffered unspecific viral infection during pregnancy, he was born at full term via an uncomplicated vaginal delivery (normal Apgar score, birth weight and length > 50th percentile). Metabolic screening was unremarkable, and there were no congenital malformations. He experienced two episodes of transient cyanosis at age $6 \mathrm{wk}$ with spontaneous resolution. Since the age of $3 \mathrm{yr}$, he has been under regular surveillance of medical experts because of global developmental delay. He was only able to sit on his own at $18 \mathrm{mo}$ and failed to walk unassisted before the age $3 \mathrm{yr}$. He had motor coordination problems, with both coarse and fine movements being very clumsy. Speech development was profoundly impaired, such that by the age of $5 \mathrm{yr}$ only a few short phrases were expressed. Over time, he was noted to have significant socialization difficulties and other behavioral abnormalities, including negativism, lack of emotional control, and autoaggressive reactions. Neuropsychological assessment at the age of $10 \mathrm{yr}$ yielded a diagnosis of moderate intellectual disability and possible autism spectrum disorder. At this age, he was able to attend special education classes and speech therapy, producing some improvements in expressive language skills.

From the age of $13 \mathrm{yr}$ onward, proband A-4's motor functions progressively deteriorated. He started to develop worsening limb coordination and difficulty walking, accompanied by the appearance of dystonic posturing of his left leg and forceful trunk retroflexion. In the beginning, involuntary movements were only occasionally observed, but within $12 \mathrm{mo}$, symptoms persisted throughout the day and had a tendency to spread. By the age of $15 \mathrm{yr}$, disabling twisting postures affected the neck, torso, and all four extremities. Additionally, he manifested irregular jerks and abnormal adventitious movements of the arms, more pronounced by intentional action, along with increasing articulation deficits. Neurological evaluation at the age of $16 \mathrm{yr}$ revealed generalized dystonia (retro torticollis, dystonia of the trunk and limbs bilaterally) combined with choreoathetoid movements aggravated by voluntary actions, hyperkinetic dysarthria, myoclonus, and minor ataxic features. Physical examination 
A

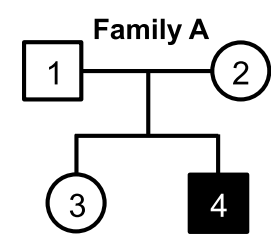

c.981+1G>A (p.Lys303Serfs $\left.{ }^{\star} 28\right)$

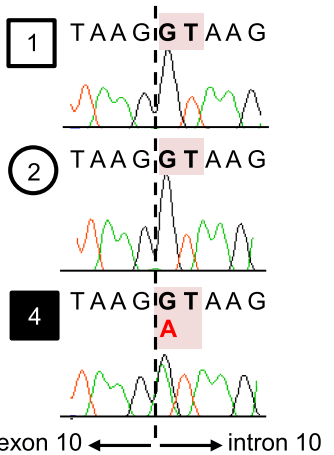

B

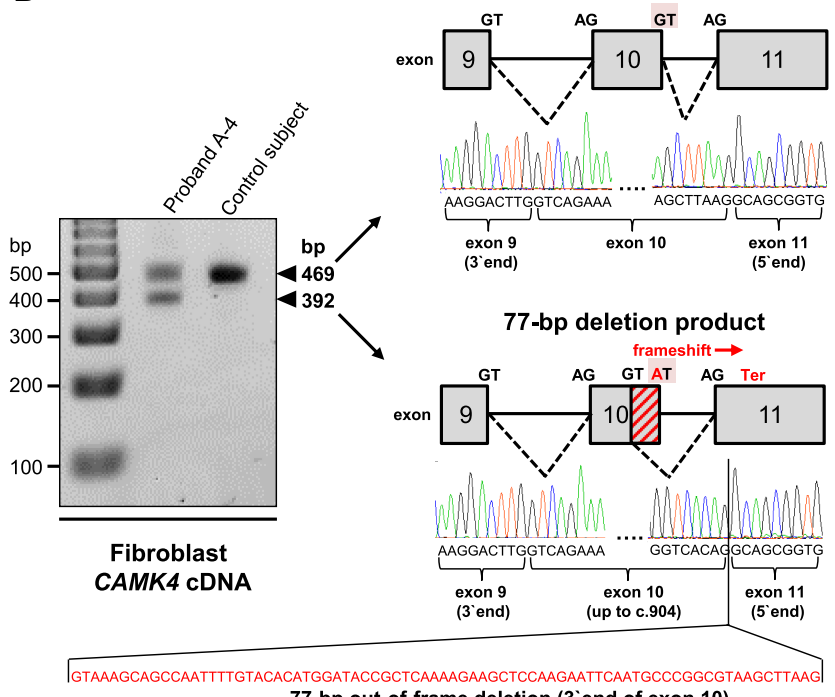

C

Chr.5q22.1

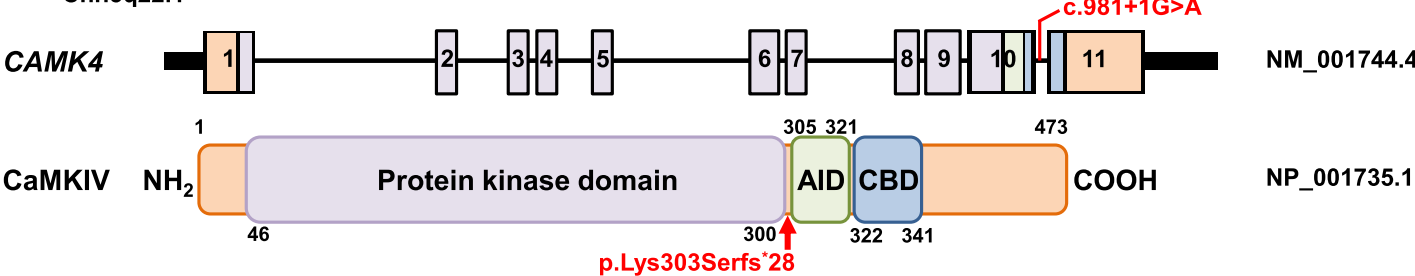

Figure 2. Identification of a de novo heterozygous essential splice-site variant, c.981+1G>A, in CAMK4 and its predicted consequences at the transcript and protein level. (A) Pedigree for the investigated family $A$ and CAMK4 splice-disrupting variant in genomic DNA. The affected individual (proband A-4) is shaded black. Sanger sequencing confirmed the de novo status of the exome-identified c.981+1G $>$ A variant located within the canonical GT splice donor of the penultimate exon 10 of CAMK4. (B) RT-PCR followed by gel electrophoresis and Sanger analysis in dermal fibroblasts from proband A-4 and a control subject. Apart from the 469-bp normal size cDNA product, proband A-4's cells produce, in equal amount, a second product of higher mobility (392 bp). Direct sequencing revealed that the 392-bp product is the result of utilization of a cryptic GT splice site upstream of the mutated donor, with generation of an abnormal mRNA isoform lacking the final $77 \mathrm{nt}$ of exon 10 (diagonally hashed rectangle), leading to a frameshift and the introduction of a premature termination codon in the last exon of CAMK4 (p.Lys303Serfs*28). WT, wild-type. (C) Diagram (not drawn to exact scale) of the human CAMK4 locus (NM_001744.4) and its encoded protein (NP_001735.1). The identified c.981+1G>A variant is shown relative to exonic structure and the position of the protein truncation (p.Lys303Serfs*28) is highlighted with a red arrow. Numbering refers to exons (upper panel) and amino acids (lower panel). Key protein domains are illustrated in color: purple for the protein kinase domain, green for the AID, and blue for the CBD.

was unremarkable and documented no gross dysmorphic stigmata. During a 12-yr follow-up, his neurologic disease course stabilized, despite some reports of intermittent worsening of baseline dystonia. On his most recent clinical examination at the age of $28 \mathrm{yr}$, proband A-4 continued to have a persistent mixed generalized hyperkinetic movement disorder, dominated by dystonia and chorea (Fig. 1). To some extent, hyperkinetic movements were superimposed with complex motor stereotypies. As a result of concomitant truncal and lower limb ataxia, he displayed unsteady gait but could walk without assistance.

Movement disorder symptoms were irresponsive to trialed medications including levodopa, anticholinergics, tiapride, and botulinum toxin. Magnetic resonance imaging (MRI) 
of the brain was notable for the presence of localized atrophy involving the cerebellum. Neither signs of leukodystrophy nor structural defects were evident in the basal ganglia, white matter, and corpus callosum. Proband A-4 had an extensive array of normal diagnostic evaluations that included electroencephalography, cerebrospinal fluid analysis, and nerve conduction studies, as well as numerous blood and/or urine biochemistry examinations (complete blood count, comprehensive metabolic panel, laboratory test for Wilson's disease, and profiling of amino acids, organic acids, triglycerides, and vitamins). Genetic testing composed of karyotyping, chromosomal microarray, and screening for DYT-TOR1A, DYTTHAP1, DYT-GCH1, spinocerebellar ataxias 1, 2, 3, 6, 7, 8, 12, 17, Friedreich's ataxia, and fragile $X$ syndrome was negative.

To comprehensively delineate proband A-4's genetic profile and uncover potentially pathogenic DNA sequence variants, he was enrolled in a research study focusing on the etiologic origins of dystonia (Technical University of Munich and Helmholtz Center Munich). Proband-parent trio whole-exome sequencing (WES) was conducted, coupled with experimental characterization of the identified candidate causative variant.

\section{Genomic Analyses}

For WES of proband A-4 and his biological parents, the average coverage depth across the exome-capture region was $161 \times$, and $98.8 \%-99.1 \%$ of the target nucleotides were covered at a read depth of $20 \times$ or greater (Table 2). The trio WES data set was first studied in a search for pathogenic or likely pathogenic variations in published genes linked to neurodevelopmental disorders, intellectual disability, or heritable movement abnormalities, but no suspicious findings were revealed. Given proband A-4's severe clinical presentation and the fact that he was the only affected individual in his family, we next hypothesized that the syndrome was caused by either rare recessive variants or an ultra-rare damaging de novo dominant event in a single yet-undescribed disease-causing gene. Scrutiny of the WES data under the assumption of a recessive mode of inheritance for the observed clinical presentation detected four compound heterozygous variants in two genes, all of which were considered to have insufficient evidence for pathogenicity (for an explanation, see Supplemental Table). Bioinformatics subtraction analysis incorporating proband A-4's and both his parents' WES variant profiles led to the identification of a single heterozygous de novo alteration, Chr 5 : g.110818636G >A (c.981+1G>A [p.?]) at CAMK4 (hg19, NM_001744.4), as an extremely interesting candidate for follow-up investigation (Tables 1 and 3). Evaluation of the full WES data sets demonstrated that CAMK4 was the only gene that contained a high-confidence de novo variant call.

The prioritized CAMK4 c.981+1G >A variant generated a GT>AT substitution in the ubiquitously conserved splice-donor dinucleotide of the penultimate exon 10 (Fig. 2A-C). The variant was unreferenced in the literature and dbSNP142 and not found in the 1000 Genomes and Genome Aggregation Database (gnomAD) data sets containing more than 248,000 population alleles. Moreover, the variant was unique in our own in-house exomes $(N=12,000)$ from individuals with a wide range of clinical diagnoses. Sanger

\begin{tabular}{|c|c|c|c|c|c|c|c|c|}
\hline $\begin{array}{l}\text { Total number } \\
\text { of reads }\end{array}$ & $\begin{array}{l}\text { Total number } \\
\text { of mapped } \\
\text { reads }\end{array}$ & $\begin{array}{l}\text { Mapped } \\
\text { reads (\%) }\end{array}$ & $\begin{array}{c}\text { Mapped } \\
\text { sequence } \\
\text { (Gb) }\end{array}$ & $\begin{array}{c}\text { Bases } \\
\text { covered } \\
\geq 20 \times(\%)\end{array}$ & $\begin{array}{l}\text { Average } \\
\text { exome } \\
\text { coverage }\end{array}$ & $\begin{array}{l}\text { Average } \\
\text { read length } \\
(b p)\end{array}$ & $\begin{array}{c}\text { Coverage at } \\
\text { CAMK4 } \\
\text { described } \\
\text { variant }\end{array}$ & $\begin{array}{c}\text { CAMK4, } \\
\text { fraction at } \\
\geq 20 \times \\
\text { coverage (\%) }\end{array}$ \\
\hline $162,220,172$ & $162,033,697$ & 99.89 & 16.37 & 99.09 & $169.94 \times$ & 101 & $\begin{array}{c}67 \times(55 \% \\
\text { alternate allele })\end{array}$ & 100 \\
\hline
\end{tabular}




\begin{tabular}{|c|c|c|c|c|c|c|c|c|c|}
\hline Gene & Chromosome & $\begin{array}{l}\text { HGVS DNA } \\
\text { reference }\end{array}$ & $\begin{array}{l}\text { HGVS protein } \\
\text { reference }\end{array}$ & $\begin{array}{l}\text { Variant } \\
\text { type }\end{array}$ & $\begin{array}{l}\text { Predicted } \\
\text { effect }\end{array}$ & $\begin{array}{l}\text { dbSNP/ } \\
\text { dbVar ID }\end{array}$ & Genotype & ClinVar ID & $\begin{array}{c}\text { Parent of } \\
\text { origin }\end{array}$ \\
\hline CAMK4 & $\begin{array}{l}\text { Chr 5: } \\
\quad 110,818,636\end{array}$ & $\begin{array}{r}\text { NM_001744.4: } \\
\text { c. } 981+1 \mathrm{G}>\mathrm{A}\end{array}$ & $\begin{array}{l}\text { NP_001735.1: } \\
\text { p.Lys303Serfs*28 }\end{array}$ & $\begin{array}{c}\text { Essential } \\
\text { splice } \\
\text { site }\end{array}$ & $\begin{array}{l}\text { Aberrant } \\
\text { splicing }\end{array}$ & $\begin{array}{l}\text { Not } \\
\quad \text { available }\end{array}$ & Heterozygous & SCV000804199 & De novo \\
\hline
\end{tabular}

sequencing of genomic DNA for proband $A-4$, his sister, and his parents proved that the c. $981+1 \mathrm{G}>\mathrm{A}$ variant had arisen de novo (Fig. 2A). Combined scores of CADD and splicedefect simulation software predicted that c. $981+1 \mathrm{G}>$ A was in the top $1 \%$ of most deleterious mutations and likely to be functionally relevant because of a disruption of CAMK4 pre-mRNA processing (Table 1; Desmet et al. 2009; Kircher et al. 2014). To directly test the latter prediction and evaluate the potential pathogenicity of the c.981+1G $>$ A variant, we synthesized cDNA from isolated RNA of fibroblast cell lines of proband A-4 and a healthy control individual. PCR amplification of cDNA was performed with a primer set targeting CAMK4 exon 7-8 junction (forward primer) and exon 11 (reverse primer). Whereas wild-type cells produced a single transcript with the expected PCR size (469 bp), proband A-4 expressed two products: the wild-type transcript and a smaller transcript of $392 \mathrm{bp}$ (Fig. 2B). Sanger analysis of the proband-specific transcript revealed an aberrant mRNA species lacking the final $77 \mathrm{nt}$ of the penultimate exon 10 because of the activation of a cryptic splice-donor site upstream of c. $981+1 \mathrm{G}>\mathrm{A}$. This deletion altered the reading frame to introduce a premature termination codon in the last exon of CAMK4, leading to a predicted chain-truncated protein: p.Lys303Serfs*28 (Fig. 2B,C). Frameshift mutations that create premature termination codons within last exons are expected to render the resulting transcripts insensitive to nonsense-mediated decay (NMD) (Kervestin and Jacobson 2012; Lindeboom et al. 2016). Therefore, we reasoned that the c.981+1G>A CAMK4 variant mRNA was not suppressed by NMD. In support of this conjecture, inspection of gel-separated RT-PCR amplicons revealed wild-type and aberrantly spliced CAMK4 transcripts in similar proportion (Fig. 2B). Furthermore, sequencing of cDNA obtained from fibroblasts of proband A-4 showed the presence of both the wild-type and the truncated transcripts, although the latter were visualized at slightly lower levels (Fig. 3A). In total, our results strongly suggested that the c.981+1G>A allele led to stable heterozygous expression of an mRNA that was expected to translate into a product p.Lys303Serfs*28. The predicted consequence of the p.Lys303Serfs*28 variant is the production of CaMKIV protein with truncation of its autoregulatory domain, but preservation of its protein kinase domain (Fig. 2C).

\section{Cellular Protein Biochemical Studies}

To examine the expression of CAMK4 c.981+1G>A (p.Lys303Serfs*28) at the protein level, we performed western blot analysis in fibroblast cell lines obtained from proband A-4 and two healthy donors. In proband-derived cells, immunoblotting yielded a CaMKIV normalsize band and a second band of $\sim 37 \mathrm{kDa}$, corresponding to p.Lys303Serfs*28, which showed reactivity with an antibody against residues 131-147 of CaMKIV (Fig. 3B; Supplemental Fig. S1). Immunoblot band intensity indicated that the expression of p.Lys $303 \mathrm{Serfs} * 28$ was lower than that of the full-length protein.

Numerous studies have shown that synthetic mutant CaMKIV species lacking the autoregulatory domain are constitutively active (Cruzalegui and Means 1993; Chatila et al. 1996; Anderson et al. 2004). Such constructs provide a powerful means of studying the consequences of up-regulated CaMKIV signaling in vitro and in vivo (Sun et al. 1994; Kane and Means 2000; See et al. 2001; Wu et al. 2002; Marie et al. 2005). To test the hypothesis that 
A

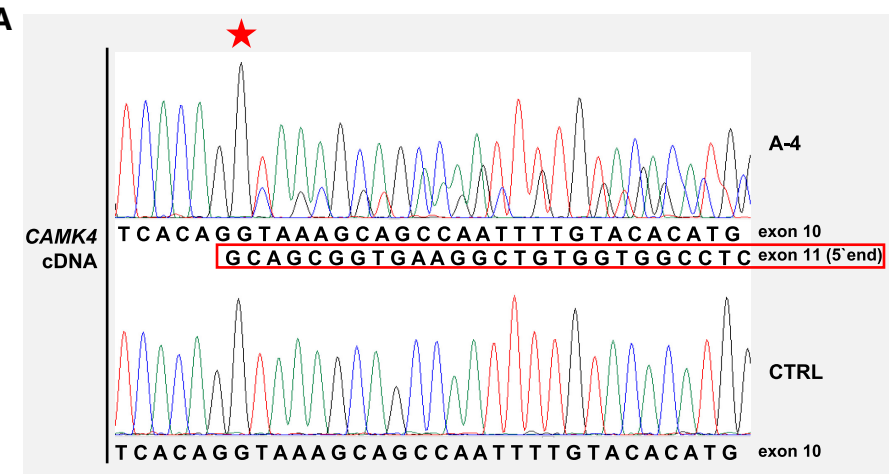

C

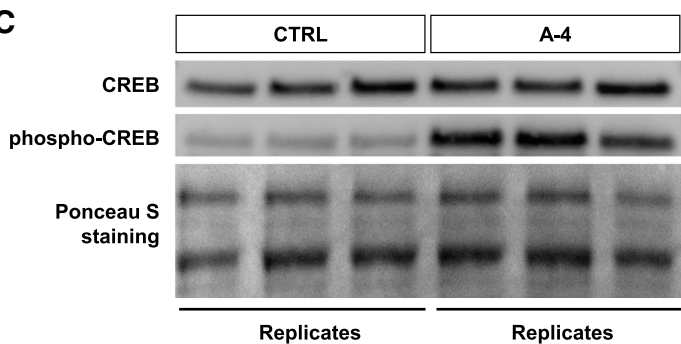

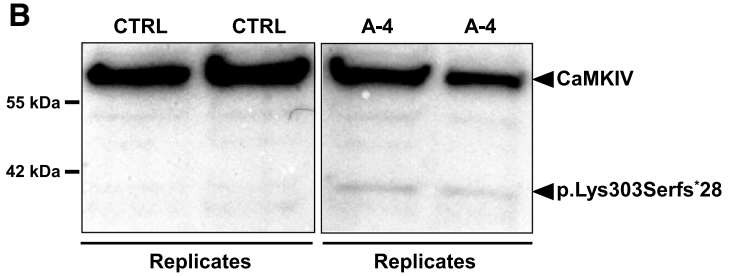

D

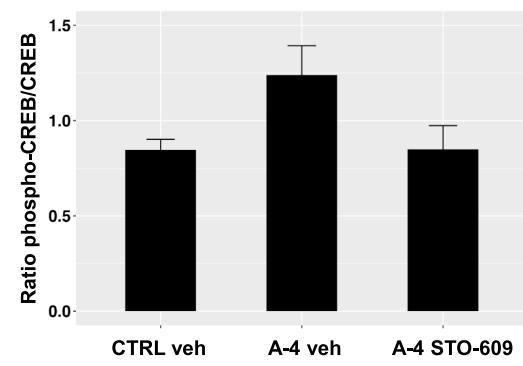

CREB

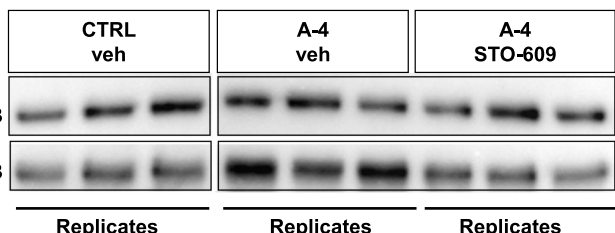

Figure 3. The CAMK4 c.981+1G>A (p.Lys303Serfs*28) allele is expressed in proband-derived cells and engenders a gain-of-function effect. (A) Sanger chromatograms of fibroblast CAMK4 cDNA from proband A-4 and a healthy control (CTRL). Proband A-4's cDNA shows wild-type and c.981+1G>A variant sequence. The nucleotide position at which the 77-bp deletion begins in proband A-4 is indicated with a star. (B) Western blot analysis using an anti-human CaMKIV antibody raised against the amino terminus of the protein. A truncated protein of $\sim 37 \mathrm{kDa}$ (p.Lys303Serfs*28) was detected in proband A-4's cells (A-4) but not in control cells (CTRL). Boxes represent cropped sections from a digital image of a single membrane (an uncropped version of the image can be found in Supplemental Fig. S1). Two biological replicates are shown for each cell line (CTRL, A-4). (C) Western blot analysis of phospho-CREB and total CREB expression in fibroblasts from proband $A-4$ (A-4) and a healthy control (CTRL). Ponceau $S$ staining was used as a loading control. Three biological replicates are shown for each cell line (CTRL, A-4). (D) (Bottom) Western blot analysis assessing phospho-CREB and total CREB expression in fibroblasts derived from proband A-4 and a healthy control (CTRL). Cells were treated with vehicle (veh) or STO-609, an inhibitor of CaMKK activity. Boxes represent cropped sections from digital images of the same membrane (uncropped versions of the images can be found in Supplemental Fig. S2). Three biological replicates are shown for each condition (CTRL veh, A-4 veh, A-4 STO-609). (Top) Bar plot showing results of densitometric quantification, depicting the ratio of phosphorylated CREB to total CREB. Data in the bar plot are presented as mean \pm standard error of the mean, $n=3$.

proband A-4's p.Lys303Serfs*28 variant also mediated enhanced activity, we assessed phosphorylation of cyclic AMP-responsive element-binding protein (CREB), a major downstream substrate of CaMKIV, in proband and control cells (Sun et al. 1994). Consistent with previously described observations in cells transfected with constitutively active CaMKIV mutants (Sun et al. 1994; Joseph and Turrigiano 2017), immunoblotting demonstrated a significant increase of CREB phosphorylation at serine residue 133 in proband-derived fibroblasts relative to controls (Fig. 3C). To confirm that this effect was mediated by CaMKIV, we treated cells with STO-609, a potent inhibitor of CaMKK, the upstream activator of CaMKIV (Tokumitsu et al. 2002). Notably, it has been demonstrated that CaMKIV lacking its autoregulatory domain still requires activation by CaMKK to adopt a constitutively active state (Chatila et al. 1996). As shown in Figure 3D (Supplemental Fig. S2), treatment of mutant cells with 
STO-609 restored phosphorylation of CREB at serine-133 to the basal control level. Collectively, our findings confirmed the production of a truncated CaMKIV protein in proband A-4 and provided experimental evidence for a c.981+1G >A (p.Lys303Serfs*28)-induced overactivation of CaMKIV signaling, which could be pharmacologically reversed by inhibition of CaMKK. We concluded that c.981+1G >A (p.Lys303Serfs*28) is a gain-of-function allele and that the effects of this variant are driven by the loss of CaMKIV's autoregulatory domain.

\section{DISCUSSION}

A central principle of precision neurology is the search for personalized therapeutic interventions on the basis of causal gene identification, molecular pathway definition, and delineation of mutation-specific pathological mechanisms. Although the vast genetic heterogeneity of neurodevelopmental disorders and congenital movement disabilities has started to be appreciated (Deciphering Developmental Disorders 2015; Carecchio and Mencacci 2017; Lohmann and Klein 2017), we continue to encounter phenotypic presentations whose molecular underpinnings remain to be untangled. In the present study, we adopted an unbiased trio WES strategy to discover a de novo heterozygous CAMK4 consensus splice-site alteration $(\mathrm{c} .981+1 \mathrm{G}>\mathrm{A})$ as the only suspicious finding in a proband who manifested a remarkable spectrum of neurodevelopmental deficits including global psychomotor delay, intellectual disability, autistic traits, adolescence-onset hyperkinetic movement disorder, ataxia, and cerebellar atrophy.

To understand the functional impact of the identified CAMK4 splicing variant, we complemented our WES approach by predictive modeling, cDNA experimentation, and protein biochemical analysis. By doing so, we were able to show that the c.981+1G>A variant generated a truncated, stable mRNA coding for an autoregulatory domain-deficient version of CaMKIV (p.Lys303Serfs*28). Further, recapitulating the effects of engineered deletion of the CaMKIV autoregulatory element (Cruzalegui and Means 1993; Sun et al. 1994), proband-derived cells demonstrated an up-regulation of CaMKIV signaling, suggesting that c.981+1G>A (p.Lys303Serfs*28) confers the encoded protein with a gain of function. Finally, we generated evidence that the gain-of-function behavior of the c.981+1G>A (p.Lys303Serfs*28) allele was sensitive to specific inhibition of CaMKIV's physiological activator CaMKK.

Multiple lines of evidence support the de novo variant in CAMK4 in this study as explanatory for the clinical syndrome of our proband. First, existing gene expression and mouse model data are consistent with a role for CAMK4 in neurodevelopmental disease. According to publicly available data repositories (The Allen Mouse Brain Atlas, The Human Protein Atlas) (Lein et al. 2007; Uhlén et al. 2015) and previous studies (Jensen et al. 1991; Nakamura et al. 1995), CaMKIV is expressed with spatial and developmental temporal specificity, with significant enrichment in the brain as compared to other tissues. In the postnatal brain, CaMKIV is highly expressed in the granule and Purkinje cells of the cerebellum, which are crucial for coordination and motor control (Ohmstede et al. 1989). In addition, high levels of CaMKIV are present in hippocampal neurons mediating essential aspects of learning and memory (Bito et al. 1996). The functional significance of these expression patterns is reflected in mice harboring a genetic defect of Camk4. Ribar and colleagues produced Camk4 null mice, which exhibited spontaneous tremulous and ataxic movements together with gross deficiencies in balance and sensorimotor performance (Ribar et al. 2000). The severe locomotor defects were accompanied by reduced numbers of Purkinje neurons, demonstrating that CaMKIV is required for normal development and function of 
COLD SPRING HARBOR Molecular Case Studies
CAMK4 variant in neurodevelopmental motor disease the cerebellum (Ribar et al. 2000). Furthermore, Wei and colleagues, studying an independent null line, showed alterations in hippocampal physiology and memory function, thereby establishing a link between Camk4 and cognitive development (Wei et al. 2002). Of note, in both studies, the modification of Camk4 was toward homozygous loss of function. Nevertheless, these observations are in good agreement with the hypothesis that a mutation of CaMKIV contributed to the cognitive and motor symptoms in our proband.

Second, the investigation of large genomic variation resources and gene-level probability scores indicates that the c.981+1G >A (p.Lys303Serfs*28) variant is a unique molecular event in a gene that is likely to be relevant to dominant non-haploinsufficient disease. Based on DOMINO, a tool for inferring the likelihood for a gene to carry dominant mutations, CAMK4 is predicted to have a very high probability of producing human autosomal dominant clinical phenotypes (Table 1; Quinodoz et al. 2017). Curiously, interrogation of 123,136 control exomes of the gnomAD database and 12,000 in-house exomes from cohorts with unrelated diseases identifies several CAMK4 protein-truncating variants (PTVs) that are expected to result in NMD of their mRNAs (Fig. 4), consistent with the prediction that CAMK4 is tolerant to heterozygous loss-of-function variation (probability of being loss-of-function intolerant [pLI] score of 0.02, as estimated by Lek et al. [2016]). In contrast, c.981+1G >A (p.Lys303Serfs*28) is the only CAMK4 PTV in our in-house exome collection predicted to encode an mRNA that evades NMD and results in a CaMKIV protein with selective depletion of its carboxy-terminal autoregulatory domain. Similarly, no such variant is registered in gnomAD (Fig. 4). Together, these findings buttress the hypothesis that CAMK4 c.981+1G >A (p.Lys303Serfs*28) is a dominant disease-relevant mutation that causes our proband's phenotype via a pathophysiological mechanism other than heterozygous loss of protein activity. This interpretation is in accordance with recent studies demonstrating that rare carboxyl-terminal PTVs can be found in dominant disease genes and that these mutations do not act through haploinsufficiency (Simpson et al. 2011; Hood et al. 2012; White et al. 2015; Jansen et al. 2017; Palmer et al. 2017). We attempted to identify additional putative CAMK4 gain-of-function variants (PTVs predicted to mediate selective loss of the autoregulatory domain of (aMKIV) in 100 internal exomes from Czech dystonia probands (Helmholtz Center Munich) and in more than 13,000 neurodevelopmental disease trio exomes available through denovo-db (Turner et al. 2017). However, no more mutations were found, implying that CAMK4-related clinical disease might be exceedingly rare.

Third, substantial amounts of available data suggest that enhancement of CaMKIV activity has detrimental neurophysiological effects. It is well known that CaMKIV is a pivotal mediator of brain activity-dependent $\mathrm{Ca}^{2+}$ dynamics in circuit development, neuronal transmission, and synaptic plasticity (Kang et al. 2001; Bading 2013). Via activation of transcription factors such as CREB, CaMKIV has long been implicated in the control of neuronal gene expression (Corcoran and Means 2001). Furthermore, a broad range of evidence has established CaMKIV as an important player in $\mathrm{Ca}^{2+}$-mediated regulation of alternative splicing (Xie and Black 2001; Xie et al. 2005). Considering these fundamental bioactivities, it is not surprising that deregulation of CaMKIV leads to disorganization of neuronal function. At the molecular level, constitutive activation of CaMKIV causes significant changes in transcription factor activities and might therefore compromise the coordinated expression of target gene programs required for brain development and neuronal homeostasis. As an example, Kane and Means have demonstrated that an activating CaMKIV mutation markedly alters transcriptional responses regulated by orphan nuclear receptors, which have well-recognized roles in the maturation of neuronal subtypes such as the cerebellar Purkinje cells (Kane and Means 2000). Interestingly, mutations of orphan nuclear receptor genes have been discovered to result in neurodevelopmental delay, intellectual disability, and cerebellar atrophy-associated motor anomalies, features also coincident with the CAMK4 mutation identified here (Guissart et al. 2018). Increased CaMKIV activity also affects the fine-tuning 


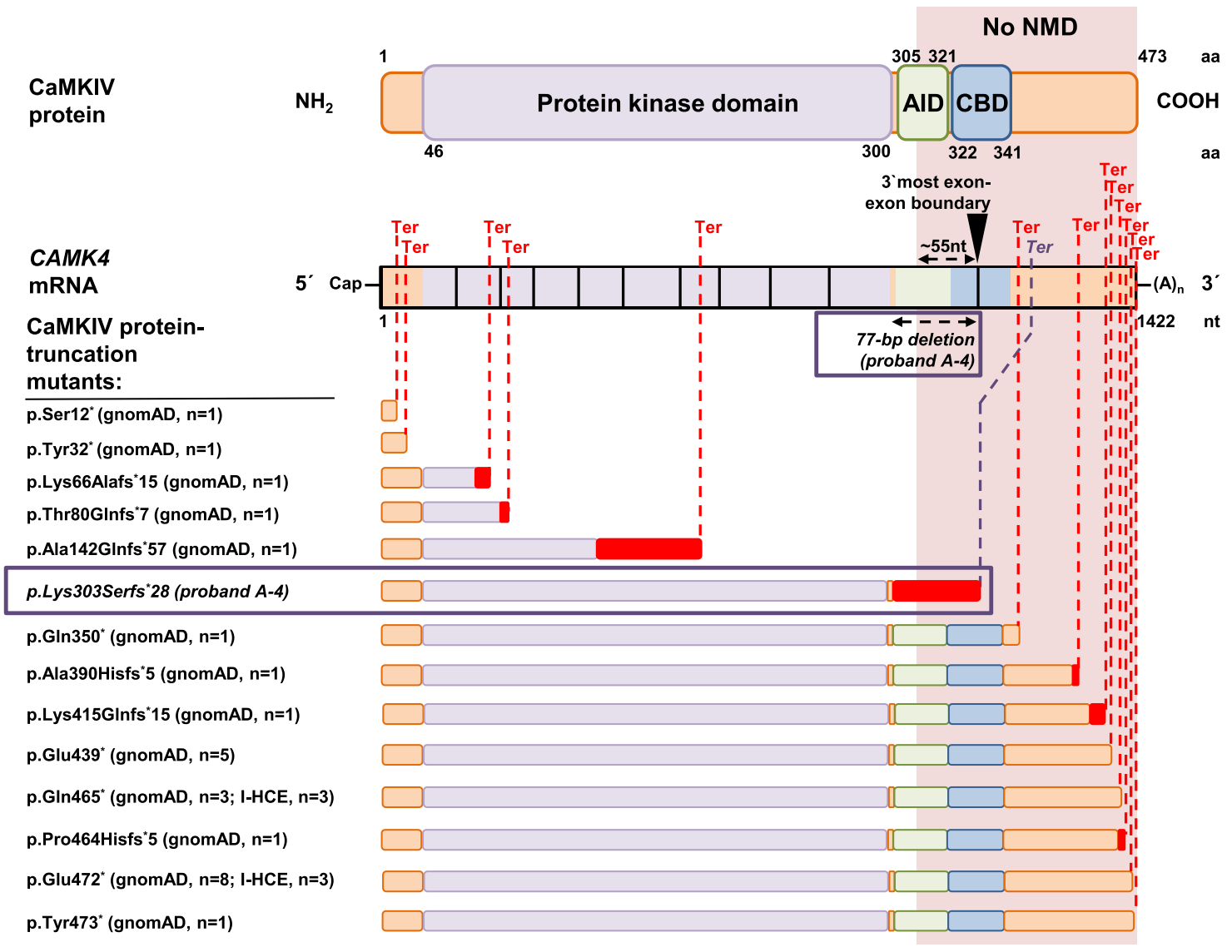

Figure 4. CAMK4 variants predicting selective abrogation of the autoregulatory domain of CaMKIV are not found in human variation databases. CAMK4 PTVs found in 123,136 control exomes of the Genome Aggregation Database (gnomAD), 12,000 in-house-sequenced control exomes (I-HCE), and proband A-4 are illustrated. Schematic representations of hypothetical and predicted expressed truncated proteins and locations of premature termination codons (Ter; dashed red lines) are depicted in relation to CAMK4 mRNA and CaMKIV domain structures. The protein kinase domain is shown in purple, the autoregulatory domain is shown in green (AID) and blue (CBD), and frameshift sequence is colored red. Amino acid (aa) and nucleotide (nt) numbers are given. Also indicated is the last exon-exon boundary (exon10-exon11, black inverted triangle) along with the $\sim 55-n t$ position upstream of this boundary. PTVs producing termination codons beyond the last $\sim 55 \mathrm{nt}$ of the penultimate exon are expected to result in truncated mRNAs that are resistant to NMD (no NMD; orange box extending through the figure). gnomAD documents 13 different PTVs (seven nonsense variants and six frameshift variants) in 26 independent exomes and our in-house exome data set two different PTVs (two nonsense variants) in six independent exomes. Of these PTVs, five are expected to cause truncation of all relevant protein domains (protein kinase domain plus autoregulatory domain) and trigger NMD. The remaining eight PTVs are expected to encode mRNAs that escape NMD but retain functionality of all relevant protein domains. Strikingly, the only exome-identified PTV that is predicted to (1) not trigger NMD and (2) cause selective abrogation of the autoregulatory domain of CaMKIV is proband A-4's p.Lys303Serfs*28 variant resulting from a 77-bp deletion in exon10 (boxed). Of note, gnomAD also contains two CAMK4 essential splice-site variants (c.387-1G>A [p.?], c.459+2dupT [p.?]), both of which are predicted to cause out-of-frame skipping of exon 5 and thus deleteriously affect the protein kinase domain (not illustrated).

of spatiotemporally controlled splicing patterns in neurons. Different studies have proven that overexpression of constitutively active CaMKIV results in an altered constitution of neuron-specific receptor and channel pre-mRNAs, including those encoding members of the NMDA-sensitive glutamate receptor family (Xie and Black 2001; Lee et al. 2007). CaMKIV 
COLD SPRING HARBOR Molecular Case Studies
CAMK4 variant in neurodevelopmental motor disease overactivation could thereby disturb the electrical properties of neurons and distort longterm neuroadaptations. At the circuit level, up-regulation of the CaMKIV signaling cascade influences homeostatic mechanisms such as global synaptic scaling (Goold and Nicoll 2010). Recent work has shown that CaMKIV gain of function leads to substantial alterations in excitatory synaptic strength, which has been suggested to predispose to neuronal circuit disorders (Joseph and Turrigiano 2017). Of particular interest in this regard, the prevailing view holds hyperkinetic motor disease, as prominently evident in our proband, to be a consequence of circuit disorganization and impaired synaptic scaling (Calabresi et al. 2016; Jinnah and Hess 2018).

Integrating all these previous observations and our present data, we propose that the CAMK4 variant we identified causes neuronal defects at both the molecular and circuit levels similar to those seen with engineered CaMKIV overactivation, which in turn might be the origin of the complex neurological phenotype presented by our proband.

In aggregate, we have described a neurological syndrome characterized by neurodevelopmental deficits evolving toward adolescence-onset hyperkinetic movement disorder. We uncovered a de novo gain-of-function variant in CAMK4 as the likely driver of this condition, thereby documenting a key role for this gene in neurodevelopment in a human subject. Our finding that pharmacological manipulation normalized CaMKIV signaling in the presence of the identified gain-of-function variant highlights the possibility of a mechanism-directed treatment strategy. Although additional studies are required, we postulate that CAMK4 gain-of-function mutation is an excellent candidate for neurodevelopmental disease causation and that therapeutic avenues addressing uncontrolled activation of CaMKIV in the brain should be explored further.

\section{METHODS}

\section{Trio WES}

Genomic DNA samples were extracted from venous blood lymphocytes of proband A-4 and his family members (parents A-1 and A-2, sister A-3) using conventional robotic techniques. Trio WES was performed on genomic DNA from proband A-4 and his parents. The full WES methodology, bioinformatic analysis, and variant interpretation protocols have been described before (Zech et al. 2016, 2017). In brief, exon targets were captured with the SureSelect All Exon system (Agilent Technologies) according to the manufacturer's recommendations. Barcoded exome libraries were pooled and sequenced using paired-end, 100-cycle chemistry on a HiSeq2500 high-throughput DNA sequencing machine (Illumina). After demultiplexing, sequences were aligned to the reference human genome (NCBI build 37 , hg19 version) with the Burrows-Wheeler Aligner and variants were called with SAMtools, Pindel, and ExomeDepth.

\section{Variant Filtering and Prioritization}

All called variants that did not pass high-confidence standards (read coverage $<10 \times$, Phredscaled quality score $<30$ ) were filtered out. Variant calls were filtered further against in-house lists of known sequencing artifacts. WES data analysis was performed using a pipeline developed in-house (Helmholtz Center Munich). De novo alterations were extracted with custom scripts comparing WES data from the parents versus the proband, as previously reported (Zech et al. 2016). Of the variants called, candidates were prioritized according to the following criteria: (1) the frequency of the alternate allele (minor allele frequency $<0.001$ [dominant variants] or $<0.005$ [recessive variants]) in control exome data sets (1000 Genomes Project, dbSNP142, in-house exome database, and gnomAD); (2) the effect on amino acid sequence; 
COLD SPRING HARBOR Molecular Case Studies
CAMK4 variant in neurodevelopmental motor disease
(3) predicted pathogenicity; and (4) cosegregation with the phenotype. We only retained rare missense, nonsense, indel, stop-loss, and splice-related intronic variants that were classified as deleterious in at least one out of three in silico prediction algorithms (PolyPhen-2, SIFT, CADD) and matched allelic state based on autosomal dominant, autosomal recessive, and $X$-linked modes of inheritance. All selected variants were inspected manually using the Integrative Genomics Viewer (IGV) and cross-referenced with the Online Mendelian Inheritance in Man (OMIM, https://www.omim.org/) and ClinVar (https://www.ncbi.nlm.nih. gov/clinvar/) databases to check for publicly available clinical and/or functional information.

\section{Confirmation of the CAMK4 Variant}

The potential disease-associated variant detected through WES was verified via standard PCR amplification and Sanger sequencing. The following primer pair targeting the altered area in genomic DNA was used: forward: 5'-TCCGTGGGTCACAGGTAAAG-3'; and reverse: 5'-AGATGAAGAACGGTAGAACTATTATTG-3'.

\section{Complementary DNA (cDNA) Studies}

We collected skin biopsies of proband A-4 and two healthy control individuals. Primary dermal fibroblasts were expanded as described earlier (Zech et al. 2015). Total fibroblast RNA was isolated by using the RNeasy kit (QIAGEN), and RNA integrity was determined using the Agilent 2100 Bioanalyzer with the use of RNA 6000 Nano chips. Reverse transcription was carried out via the SuperScript First-Strand Synthesis System for RT-PCR (Invitrogen) using $1000 \mathrm{ng}$ of total RNA as a template. The primer pair for PCR of fibroblast-derived cDNA was designed to encompass CAMK4 exons 9 and 10-forward: 5'-GGGTACTGCGCACCTG AAAT-3' (in CAMK4 exon 7-8 junction); and reverse: 5'-TTGGAGAAGGGTCTCGGCTA-3' (in CAMK4 exon 11). PCR products were electrophoretically separated on $2 \%$ agarose gels and visualized by Midori Green staining. Separated PCR products were extracted and purified from agarose gel using the QIAquick Gel Extraction Kit (QIAGEN). Sequence determination of cDNA amplicons was done by Sanger analysis using the cDNA primer set described above.

\section{Western Blot Analysis in Fibroblasts}

Fibroblasts of proband A-4 and two healthy donors were maintained in Dulbecco's Modified Eagle Medium (DMEM) supplemented with 10\% fetal bovine serum, $50 \mathrm{U} / \mathrm{ml}$ penicillin, and $50 \mathrm{~g} / \mathrm{ml}$ streptomycin. Near-confluent fibroblasts were treated with STO-609 (5 $\mu \mathrm{M}$; Merck) or vehicle (DMSO). Two hours later, lysates were prepared with lysis buffer $(150 \mathrm{mM} \mathrm{NaCl}$, 1\% NP-40, $50 \mathrm{mM}$ Tris, $\mathrm{pH}$ 8, protease inhibitors [cOmplete Mini, Merck]), followed by agitation and centrifugation. Supernatants were subjected to SDS-PAGE and semidry immunoblotting. Primary antibodies used (all rabbit, 1:1000) were anti-CaMKIV (ThermoFisher PA1-542), anti-CREB (Cell Signaling 9197), and anti-phospho-CREB (Cell Signaling 9198). Secondary antibody was HRP-conjugated donkey anti-rabbit IgG (BioLegend 406401). Chemiluminescent detection was performed with ECL Prime (Amersham). Protein abundance was quantified by signal density (ImageJ).

\section{ADDITIONAL INFORMATION}

\section{Data Deposition and Access}

Whole-exome sequencing data are not publicly available because consent could not be obtained. The CAMK4 variant found in this study has been submitted to ClinVar (http://www. ncbi.nlm.nih.gov/clinvar/) under accession number SCV000804199. 
C OLD SPRING HARBOR Molecular Case Studies
CAMK4 variant in neurodevelopmental motor disease
Competing Interest Statement

The authors have declared no competing interest.

Received June 25, 2018; accepted in revised form September 4, 2018.

\section{Ethics Statement}

The study was approved by the local ethics review boards at Technical University of Munich, Germany, and Charles University in Prague, Czech Republic. Written informed consent was obtained from the proband and proband's parents for publication and accompanying images.

\section{Acknowledgments}

We are grateful to the proband and his family for their participation in this study. M.Z. was supported by an internal research program at Helmholtz Center Munich, Germany ("Physician Scientists for Groundbreaking Projects").

\section{Author Contributions}

M.Z. and D.D.L. conceived the study, generated and analyzed the data, and drafted and critically reviewed the manuscript. S.W. generated and analyzed the data and critically reviewed the manuscript. R.B. and T.M.S. generated the data and critically reviewed the manuscript. K.P., P.H., A.F., and E.R. provided the clinical data and critically reviewed the manuscript. R.J. conceived the study, provided the clinical data, and critically reviewed the manuscript. J.W. conceived the study, analyzed the data, and drafted and critically reviewed the manuscript.

\section{Funding}

This study was funded by the Czech Science Foundation (GACR16-13323S) as well as inhouse institutional funding from Technische Universität München, Munich, Germany; Helmholtz Zentrum München, Munich, Germany, and Charles University, Prague, Czech Republic (PROGRES Q27). D.D.L. was supported by DFG grant LA 3830/1-1.

\section{REFERENCES}

Anderson KA, Noeldner PK, Reece K, Wadzinski BE, Means AR. 2004. Regulation and function of the calcium/ calmodulin-dependent protein kinase IV/protein serine/threonine phosphatase 2A signaling complex. J Biol Chem 279: 31708-31716.

Bading H. 2013. Nuclear calcium signalling in the regulation of brain function. Nat Rev Neurosci 14: 593-608.

Bito H, Deisseroth K, Tsien RW. 1996. CREB phosphorylation and dephosphorylation: $\mathrm{Ca}^{2+}$ - and stimulus duration-dependent switch for hippocampal gene expression. Cell 87: 1203-1214.

Calabresi P, Pisani A, Rothwell J, Ghiglieri V, Obeso JA, Picconi B. 2016. Hyperkinetic disorders and loss of synaptic downscaling. Nat Neurosci 19: 868-875.

Carecchio M, Mencacci NE. 2017. Emerging monogenic complex hyperkinetic disorders. Curr Neurol Neurosci Rep 17: 97.

Chatila T, Anderson KA, Ho N, Means AR. 1996. A unique phosphorylation-dependent mechanism for the activation of $\mathrm{Ca}^{2+} /$ calmodulin-dependent protein kinase type IV/GR. J Biol Chem 271: 21542-21548.

Corcoran EE, Means AR. 2001. Defining $\mathrm{Ca}^{2+} /$ calmodulin-dependent protein kinase cascades in transcriptional regulation. J Biol Chem 276: 2975-2978.

Cruzalegui FH, Means AR. 1993. Biochemical characterization of the multifunctional $\mathrm{Ca}^{2+} /$ calmodulin- $^{2}$ dependent protein kinase type IV expressed in insect cells. J Biol Chem 268: 26171-26178.

Deciphering Developmental Disorders Study. 2015. Large-scale discovery of novel genetic causes of developmental disorders. Nature 519: 223-228.

Desmet FO, Hamroun D, Lalande M, Collod-Béroud G, Claustres M, Béroud C. 2009. Human Splicing Finder: an online bioinformatics tool to predict splicing signals. Nucleic Acids Res 37: e67.

Goold CP, Nicoll RA. 2010. Single-cell optogenetic excitation drives homeostatic synaptic depression. Neuron 68: 512-528.

Guissart C, Latypova X, Rollier P, Khan TN, Stamberger H, McWalter K, Cho MT, Kjaergaard S, Weckhuysen S, Lesca G, et al. 2018. Dual molecular effects of dominant RORA mutations cause two variants of syndromic intellectual disability with either autism or cerebellar ataxia. Am J Hum Genet 102: 744-759. 
Hood RL, Lines MA, Nikkel SM, Schwartzentruber J, Beaulieu C, Nowaczyk MJ, Allanson J, Kim CA, Wieczorek D, Moilanen JS, et al. 2012. Mutations in SRCAP, encoding SNF2-related CREBBP activator protein, cause Floating-Harbor syndrome. Am J Hum Genet 90: 308-313.

Jansen S, Geuer S, Pfundt R, Brough R, Ghongane P, Herkert JC, Marco EJ, Willemsen MH, Kleefstra T, Hannibal M, et al. 2017. De novo truncating mutations in the last and penultimate exons of PPM1D cause an intellectual disability syndrome. Am J Hum Genet 100: 650-658.

Jensen KF, Ohmstede CA, Fisher RS, Sahyoun N. 1991. Nuclear and axonal localization of $\mathrm{Ca}^{2+} / \mathrm{calmodulin}^{-}$ dependent protein kinase type $\mathrm{Gr}$ in rat cerebellar cortex. Proc Natl Acad Sci 88: 2850-2853.

Jinnah HA, Hess EJ. 2018. Evolving concepts in the pathogenesis of dystonia. Parkinsonism Relat Disord 46: S62-S65.

Joseph A, Turrigiano GG. 2017. All for one but not one for all: excitatory synaptic scaling and intrinsic excitability are coregulated by CaMKIV, whereas inhibitory synaptic scaling is under independent control. $J$ Neurosci 37: 6778-6785.

Kane CD, Means AR. 2000. Activation of orphan receptor-mediated transcription by $\mathrm{Ca}^{2+} /$ calmodulin- $^{2}$ dependent protein kinase IV. EMBO J 19: 691-701.

Kang H, Sun LD, Atkins CM, Soderling TR, Wilson MA, Tonegawa S. 2001. An important role of neural activitydependent CaMKIV signaling in the consolidation of long-term memory. Cell 106: 771-783.

Kervestin S, Jacobson A. 2012. NMD: a multifaceted response to premature translational termination. Nat Rev Mol Cell Biol 13: 700-712.

Kircher M, Witten DM, Jain P, O'Roak BJ, Cooper GM, Shendure J. 2014. A general framework for estimating the relative pathogenicity of human genetic variants. Nat Genet 46: 310-315.

Küry S, van Woerden GM, Besnard T, Proietti Onori M, Latypova X, Towne MC, Cho MT, Prescott TE, Ploeg MA, Sanders S, et al. 2017. De novo mutations in protein kinase genes CAMK2A and CAMK2B cause intellectual disability. Am J Hum Genet 101: 768-788.

Lee JA, Xing Y, Nguyen D, Xie J, Lee CJ, Black DL. 2007. Depolarization and CaM kinase IV modulate NMDA receptor splicing through two essential RNA elements. PLoS Biol 5: e40.

Lein ES, Hawrylycz MJ, Ao N, Ayres M, Bensinger A, Bernard A, Boe AF, Boguski MS, Brockway KS, Byrnes EJ, et al. 2007. Genome-wide atlas of gene expression in the adult mouse brain. Nature 445: 168-176.

Lek M, Karczewski KJ, Minikel EV, Samocha KE, Banks E, Fennell T, O'Donnell-Luria AH, Ware JS, Hill AJ, Cummings BB, et al. 2016. Analysis of protein-coding genetic variation in 60,706 humans. Nature 536: 285-291.

Lindeboom RG, Supek F, Lehner B. 2016. The rules and impact of nonsense-mediated mRNA decay in human cancers. Nat Genet 48: 1112-1118.

Lohmann K, Klein C. 2017. Update on the genetics of dystonia. Curr Neurol Neurosci Rep 17: 26.

Marie H, Morishita W, Yu X, Calakos N, Malenka RC. 2005. Generation of silent synapses by acute in vivo expression of CaMKIV and CREB. Neuron 45: 741-752.

Nakamura Y, Okuno S, Sato F, Fujisawa H. 1995. An immunohistochemical study of $\mathrm{Ca}^{2+} /$ calmodulin-dependent protein kinase IV in the rat central nervous system: light and electron microscopic observations. Neuroscience 68: 181-194.

$\mathrm{Naz} \mathrm{H}$, Islam A, Ahmad F, Hassan MI. 2016. Calcium/calmodulin-dependent protein kinase IV: a multifunctional enzyme and potential therapeutic target. Prog Biophys Mol Biol 121: 54-65.

Ohmstede CA, Jensen KF, Sahyoun NE. 1989. $\mathrm{Ca}^{2+} /$ calmodulin-dependent protein kinase enriched in cerebellar granule cells. Identification of a novel neuronal calmodulin-dependent protein kinase. $J$ Biol Chem 264: 5866-5875.

Palmer EE, Kumar R, Gordon CT, Shaw M, Hubert L, Carroll R, Rio M, Murray L, Leffler M, Dudding-Byth T, et al. 2017. A recurrent de novo nonsense variant in ZSWIM6 results in severe intellectual disability without frontonasal or limb malformations. Am J Hum Genet 101: 995-1005.

Penzes P, Cahill ME, Jones KA, Srivastava DP. 2008. Convergent CaMK and RacGEF signals control dendritic structure and function. Trends Cell Biol 18: 405-413.

Quinodoz M, Royer-Bertrand B, Cisarova K, Di Gioia SA, Superti-Furga A, Rivolta C. 2017. DOMINO: using machine learning to predict genes associated with dominant disorders. Am J Hum Genet 101: 623-629.

Ribar TJ, Rodriguiz RM, Khiroug L, Wetsel WC, Augustine GJ, Means AR. 2000. Cerebellar defects in $\mathrm{Ca}^{2+} / \mathrm{cal}_{-}$ modulin kinase IV-deficient mice. J Neurosci 20: RC107.

Rosenberg SS, Spitzer NC. 2011. Calcium signaling in neuronal development. Cold Spring Harb Perspect Biol 3: a004259.

See V, Boutillier AL, Bito H, Loeffler JP. 2001. Calcium/calmodulin-dependent protein kinase type IV (CaMKIV) inhibits apoptosis induced by potassium deprivation in cerebellar granule neurons. FASEB J 15: 134-144.

Simpson MA, Irving MD, Asilmaz E, Gray MJ, Dafou D, Elmslie FV, Mansour S, Holder SE, Brain CE, Burton BK, et al. 2011. Mutations in NOTCH2 cause Hajdu-Cheney syndrome, a disorder of severe and progressive bone loss. Nat Genet 43: 303-305. 
C OLD SPRING HARBOR Molecular Case Studies
CAMK4 variant in neurodevelopmental motor disease

Sun P, Enslen H, Myung PS, Maurer RA. 1994. Differential activation of CREB by $\mathrm{Ca}^{2+} /$ calmodulin-dependent protein kinases type II and type IV involves phosphorylation of a site that negatively regulates activity. Genes Dev 8: 2527-2539.

Swulius MT, Waxham MN. 2008. $\mathrm{Ca}^{2+} /$ calmodulin-dependent protein kinases. Cell Mol Life Sci 65: 2637-2657.

Takemoto-Kimura S, Suzuki K, Horigane SI, Kamijo S, Inoue M, Sakamoto M, Fujii H, Bito H. 2017. Calmodulin kinases: essential regulators in health and disease. J Neurochem 141: 808-818.

Tokumitsu H, Wayman GA, Muramatsu M, Soderling TR. 1997. Calcium/calmodulin-dependent protein kinase kinase: identification of regulatory domains. Biochemistry 36: 12823-12827.

Tokumitsu H, Inuzuka H, Ishikawa Y, Ikeda M, Saji I, Kobayashi R. 2002. STO-609, a specific inhibitor of the $\mathrm{Ca}^{2+} /$ calmodulin-dependent protein kinase kinase. J Biol Chem 277: 15813-15818.

Turner TN, Yi Q, Krumm N, Huddleston J, Hoekzema K, F Stessman HA, Doebley AL, Bernier RA, Nickerson DA, Eichler EE. 2017. denovo-db: a compendium of human de novo variants. Nucleic Acids Res 45: D804-D811.

Uhlén M, Fagerberg L, Hallström BM, Lindskog C, Oksvold P, Mardinoglu A, Sivertsson A, Kampf C, Sjöstedt E, Asplund A, et al. 2015. Proteomics. Tissue-based map of the human proteome. Science 347: 1260419.

Wei F, Qiu CS, Liauw J, Robinson DA, Ho N, Chatila T, Zhuo M. 2002. Calcium calmodulin-dependent protein kinase IV is required for fear memory. Nat Neurosci 5: 573-579.

White J, Mazzeu JF, Hoischen A, Jhangiani SN, Gambin T, Alcino MC, Penney S, Saraiva JM, Hove H, Skovby F, et al. 2015. DVL1 frameshift mutations clustering in the penultimate exon cause autosomal-dominant Robinow syndrome. Am J Hum Genet 96: 612-622.

Wu H, Kanatous SB, Thurmond FA, Gallardo T, Isotani E, Bassel-Duby R, Williams RS. 2002. Regulation of mitochondrial biogenesis in skeletal muscle by CaMK. Science 296: 349-352.

Xie J, Black DL. 2001. A CaMK IV responsive RNA element mediates depolarization-induced alternative splicing of ion channels. Nature 410: 936-939.

Xie J, Jan C, Stoilov P, Park J, Black DL. 2005. A consensus CaMK IV-responsive RNA sequence mediates regulation of alternative exons in neurons. RNA 11: 1825-1834.

Zech M, Lam DD, Francescatto L, Schormair B, Salminen AV, Jochim A, Wieland T, Lichtner P, Peters A, Gieger $\mathrm{C}$, et al. 2015. Recessive mutations in the $\alpha 3(\mathrm{VI})$ collagen gene COL6A3 cause early-onset isolated dystonia. Am J Hum Genet 96: 883-893.

Zech M, Boesch S, Maier EM, Borggraefe I, Vill K, Laccone F, Pilshofer V, Ceballos-Baumann A, Alhaddad B, Berutti $R$, et al. 2016. Haploinsufficiency of KMT2B, encoding the lysine-specific histone methyltransferase 2B, results in early-onset generalized dystonia. Am J Hum Genet 99: 1377-1387.

Zech M, Boesch S, Jochim A, Weber S, Meindl T, Schormair B, Wieland T, Lunetta C, Sansone V, Messner M, et al. 2017. Clinical exome sequencing in early-onset generalized dystonia and large-scale resequencing follow-up. Mov Disord 32: 549-559. 


\section{COLD SPRING HARBOR Molecular Case Studies}

\section{A unique de novo gain-of-function variant in CAMK4 associated with intellectual disability and hyperkinetic movement disorder}

Michael Zech, Daniel D. Lam, Sandrina Weber, et al.

Cold Spring Harb Mol Case Stud 2018, 4: a003293 originally published online September 27, 2018 Access the most recent version at doi: $10.1101 / \mathrm{mcs} .0003293$
Supplementary http://molecularcasestudies.cshlp.org/content/suppl/2018/11/05/mcs.a003293.D Material C1

References This article cites 53 articles, 15 of which can be accessed free at: http://molecularcasestudies.cshlp.org/content/4/6/a003293.full.html\#ref-list-1

License This article is distributed under the terms of the Creative Commons Attribution License, which permits unrestricted reuse and redistribution provided that the original author and source are credited.

Email Alerting Receive free email alerts when new articles cite this article - sign up in the box at the Service top right corner of the article or click here. 